\title{
Treinamento de marcha, cardiorrespiratório e muscular após acidente vascular encefálico: estratégias, dosagens e desfechos
}

\author{
Gait training, cardiorespiratory training and strength training after stroke: \\ strategies, dose and outcomes
}

\author{
Angélica Cristiane Ovando ${ }^{[a]}$, Stella Maris Michaelsen ${ }^{[b]}$, Jonathan Ache Dias ${ }^{[c]}$, Vanessa Herber ${ }^{[\mathrm{d}]}$ \\ [a] Mestre em Ciências do Movimento Humano, Laboratório de Controle Motor (LADECOM), Programa de Pós-Graduação \\ em Ciências do Movimento Humano, Universidade do Estado de Santa Catariana (UDESC), Florianópolis, SC - Brasil, e-mail: \\ angecris@yahoo.com.br \\ [b] Doutora, docente do Curso de Fisioterapia e do Programa de Pós-Graduação em Ciências do Movimento Humano, Universidade \\ do Estado de Santa Catariana (UDESC), Coordenadora do Laboratório de Controle Motor (LADECOM), Florianópolis, SC - \\ Brasil, e-mail: michaelsenstella@hotmail.com \\ [c] Mestre em Ciências do Movimento Humano, Laboratório de Instrumentação (LABIN), Programa de Pós-Graduação em Ciências \\ do Movimento Humano, Universidade do Estado de Santa Catariana (UDESC), Florianópolis, SC - Brasil, e-mail: jonathanache@, \\ gmail.com \\ [d] Mestre em Ciências do Movimento Humano, Laboratório de Controle Motor (LADECOM), Programa de Pós-Graduação \\ em Ciências do Movimento Humano, Universidade do Estado de Santa Catariana (UDESC), Florianópolis, SC - Brasil, e-mail: \\ vane_herber@yahoo.com.br
}

\section{Resumo}

Introdução: Um número crescente de programas de treinamento com resultados positivos tem sido proposto para a reabilitação de pacientes com sequelas motoras após acidente vascular encefálico (AVE). No entanto, observa-se que muitos não oferecem recomendações no que diz respeito a indicações para técnicas e procedimentos específicos. Objetivo: Revisar a literatura pertinente sobre programas de treinamento envolvendo marcha, condicionamento cardiorrespiratório e fortalecimento muscular de membros inferiores em pacientes portadores de hemiparesia por sequela de AVE, e descrever a eficácia, limitações e efeitos desses programas na recuperação cardiovascular, funcional e motora dessa população. Método: Foi realizada uma busca por ensaios clínicos, trabalhos pré-experimentais, meta-análises e revisões de literatura que abordassem os temas treinamento físico, fortalecimento muscular, treinamento de marcha e programas de exercícios para membros inferiores após AVE. Resultados: Foram encontrados 27 artigos relatando diversos protocolos de treinamento (marcha, treinamento cardiovascular, fortalecimento muscular, entre outros) e seus efeitos no sistema cardiovascular, músculo-esquelético e sobre o 
status funcional em indivíduos portadores de hemiparesia após AVE. Conclusão: Praticamente todas as intervenções relatam resultados positivos em termos de ganhos funcionais, além de efeitos específicos de acordo com o tipo de treinamento. No entanto, as diferenças metodológicas, a carência de grupo controle em alguns estudos, a variabilidade da população estudada e os critérios de análise nem sempre permitem a recomendação segura de procedimentos específicos na prática clínica.

Palavras-chave: Acidente vascular encefálico. Reabilitação. Marcha. Força muscular. Aptidão física.

\begin{abstract}
Introduction: An increasing number of training programs with positive results has been proposed for rehabilitation of stroke survivors with motor impairments. However, few studies offer recommendations regarding indications for specific techniques and procedures. Objective: To review data from the literature about training and exercise programs in patients with hemiparesis following stroke regarding walking training, lower limb strengthening and cardiorespiratory fitness, and to describe the efficacy, limitations and effect of such programs on cardiovascular, motor recovery and functional status. Method: It was made a search in relevant medical journals for articles of clinical trials, meta-analyses, and literature reviews pertaining to physical training, muscular strengthening, gait training and exercise programs for lower limbs after stroke. Results: It was found 27 articles reporting on various training and exercise techniques (gait training, cardiovascular training, muscle strengthening, and others) and their results on the cardiovascular, musculoskeletal, and neurological systems, as well as functional status in stroke patients. Conclusion: Training and exercise programs have value in stroke rehabilitation, and published results are, in general, promising. The differences in the populations tested, methods, and criteria for analysis do not always allow the recommendation of specific procedures in clinical practice.
\end{abstract}

Keywords: Stroke. Rehabilitation. Gait. Muscular strength. Physical fitness.

\title{
Introdução
}

O acidente vascular encefálico (AVE) é a doença vascular que mais acomete o sistema nervoso central, sendo a principal causa de incapacidades físicas e cognitivas em adultos (1). No Brasil, dos indivíduos que sofreram AVE, 30\% necessitam de auxílio para caminhar e 20\% ficam com sequelas graves e incapacitantes (2).

As consequências do AVE incluem não somente danos residuais motores e cognitivos, mas também uma reduzida capacidade de suportar esforços, sendo que os principais problemas relatados são confinamento, imobilidade, perda de habilidades funcionais em função de déficit motor e, frequentemente, comorbidades metabólicas e cardiovasculares. Secundariamente, a perda ou redução da capacidade física geram um decréscimo na intensidade e frequência da prática de atividades físicas que envolvam ações da vida diária, lazer (esporte) ou trabalho (3). Recuperar a habilidade de locomover-se está entre uma das maiores preocupações de sujeitos com hemiplegia/hemiparesia, uma vez que a locomoção permite independência e tem um impacto direto no retorno ao lar (4).

Existe hoje um grande número de abordagens para o tratamento fisioterapêutico após um AVE. Desde os anos 80, tem se destacado a importância da aprendizagem motora, particularmente por meio da abordagem da aprendizagem ou reaprendizagem motora (5), que propõe a prática ativa de tarefas motoras contexto-específicas com apropriado feedback. Além disso, tem sido enfatizada a necessidade de basear a fisioterapia neurológica em pesquisa cientifica em áreas relevantes, tais como a neurociência, biomecânica, fisiologia do exercício e na testagem dos resultados para que se desenvolva uma fisioterapia baseada em evidência.

A reabilitação do paciente com sequela de AVE envolve primariamente a fisioterapia convencional, entretanto, outras técnicas foram propostas recentemente para melhorar a capacidade de suportar esforços, o desempenho da marcha, ou ainda, as habilidades físicas gerais. Alguns exemplos incluem a caminhada em 
esteira, com ou sem suspensão de peso corporal, o treinamento aeróbico e o fortalecimento muscular. Tais técnicas têm o objetivo de melhorar o desempenho funcional na marcha, e também contribuem para uma melhora geral na capacidade física. Para otimizar o tratamento de indivíduos com sequelas de AVE, é necessário avaliar os efeitos de diferentes programas de treinamento que tenham por objetivo melhorar as funcionalidade, e principalmente a competência para deambular.

Como a maioria dos pacientes acometidos por AVE apresenta problemas na deambulação $(6,7)$, condicionamento aeróbico deficitário (8) e comprometimentos musculares (9), que podem prejudicar o seu desempenho nas atividades funcionais, este trabalho propõe-se a realizar uma revisão dos estudos que têm sido realizados sobre programas de treinamento envolvendo marcha, condicionamento cardiorrespiratório e fortalecimento muscular de membros inferiores em pacientes portadores de hemiparesia por sequela de AVE. Pretende-se, assim, identificar a aplicação e indicação dos treinamentos encontrados em populações específicas de portadores de hemiparesia (sujeitos agudos, crônicos, com diferentes comprometimentos motores), além de verificar quais os desfechos que tiveram resultados positivos com o uso dessas técnicas. Pretende-se ainda identificar na literatura as características específicas dos protocolos (especificidade, duração, intensidade).

\section{Método}

Os artigos desta revisão foram selecionados a partir das bases de dados Science Direct, PubMed, SpringerLink, LILACS, Informaworld e IngentaConnect, no período entre julho e dezembro de 2008. Foram considerados para esta revisão apenas os artigos publicados na íntegra entre os anos 1980 e 2008, que foram pesquisados a partir da combinação dos termos ingleses: stroke, hemiplegia, hemiparesis, physical exercise, physical training, strength training, muscular training, exercise training, body-weight supported treadmill training, treadmill training, gait training, walking, functional activity; e em português, dos termos: acidente vascular cerebral/encefálico, hemiparesia, hemiplegia com treinamento, marcha, força muscular, treinamento em esteira e atividade funcional. Os artigos deveriam relatar ensaios clínicos randomizados ou então estudos pré-experimentais ou quasi-experimentais que envolvessem treinamentos com objetivo de melhorar a marcha, a força, o condicionamento cardiorrespiratório e a independência funcional dos indivíduos acometidos por AVE. Foram incluídos 27 trabalhos escritos com este perfil, além de dois artigos de revisão, todos na língua inglesa. Os demais artigos citados no presente estudo foram utilizados para embasar as discussões referentes aos achados dos estudos incluídos.

\section{Resultados}

\section{Treinamento de marcha}

Em geral, mais da metade dos pacientes não deambula independentemente na fase aguda após um AVE, e esta dependência para marcha pode ser observada em $25 \%$ dos pacientes três meses após o evento (6). De acordo com Goldie et al. (7), 60\% a 70\% dos indivíduos que sofrem um AVE recuperam a capacidade de marcha, embora apenas 7\% dos pacientes subagudos tenham capacidade suficiente de deambular de forma independente fora de casa. A recuperação da marcha é a meta principal na reabilitação desses pacientes. Os programas de treinamento de marcha têm como objetivo restabelecer a marcha funcional e também melhorar a qualidade ou desempenho desta.

A esteira com suspensão de peso corporal (SPC) tem sido utilizada largamente para recuperar a função locomotora após um AVE. Existe hoje uma grande variedade de estudos investigando programas de treinamento da marcha, frequentemente realizados em uma esteira com ou sem SPC.

O treinamento em esteira está fundamentado em dois princípios neurofisiológicos: a melhora da modulação do reflexo da marcha e o treinamento locomotor, envolvendo um grande volume de repetições da 
Ovando AC, Michaelsen SM, Dias JA, Herber V.

tarefa (10). O uso de esteira (com ou sem suspensão) permite que um grande número de passos seja executado dentro de uma sessão de treinamento, aumentando a prática da tarefa específica. Pacientes com sequelas por AVE podem praticar até 1.000 passos em 20 minutos de prática em esteira, comparados com apenas 50 ou 100 passos durante o mesmo período de uma sessão de fisioterapia convencional (11). O sistema com SPC oferece condições favoráveis para a recuperação da marcha, uma vez que prolonga a fase de apoio no membro plégico, melhora a simetria do passo, diminui a espasticidade dos plantiflexores do tornozelo e permite um modelo de ativação muscular mais regular, quando comparado com a caminhada no solo (12).

O treinamento em esteira com SPC permite que pacientes incapazes de deambular livremente pratiquem ciclos de marcha completa repetidamente. Visintin et al. (13) relataram que a terapia em esteira com SPC foi mais efetiva do que sem SPC em pacientes hemiparéticos subagudos, e a terapia em esteira também mostrou vantagens sobre o treino de marcha convencional, no que diz respeito ao condicionamento físico (14) e habilidade da marcha (15). No entanto, dois ensaios clínicos randomizados com indivíduos com hemiparesia aguda não conseguiram demonstrar a superioridade do treinamento com esteira, em comparação com a fisioterapia convencional, que esteve concentrada em treino de marcha no solo $(16,17)$.

Diversos protocolos para o treinamento de marcha têm sido propostos, com uma variedade de velocidades, intensidades, percentual de peso corporal suspenso, duração e também enfocando diferentes fases do AVE, com pacientes agudos e crônicos. Na maioria dos estudos, os indivíduos deveriam ser capazes de caminhar pelo menos 10 metros para serem incluídos, sendo excluídos aqueles com comorbidades cardiovasculares graves ou déficits cognitivos. Os programas eram individualizados, geralmente seguindo uma avaliação inicial, e os parâmetros de exercício e ajustes da esteira foram adaptados durante o programa. As medidas de desfecho avaliadas variaram entre os estudos. A velocidade de marcha foi um dos parâmetros mais importantes, sendo que todos os estudos avaliaram a velocidade de marcha em uma distância curta, embora os protocolos utilizados tenham variado entre os estudos, com distâncias de 3 a 10 metros, ou ainda a distância percorrida durante dois minutos (16) .

Dentre os estudos envolvendo treinamento de marcha com SPC selecionados para esta revisão, sete avaliaram a resistência para a marcha $(13,16,18-22)$, seis estudos utilizaram uma escala funcional (por exemplo, a Functional Ambulation Category - FAC) para classificar a dependência funcional da marcha (13, 15, 17, 19, $23,24)$, e quatro estudos avaliaram as atividades de vida diária $(16,17,19,25)$. Quanto ao tipo de comparação que foi utilizada nos estudos selecionados, todos compararam o treinamento de marcha em esteira com algum outro tipo de intervenção fisioterapêutica. Seis estudos compararam treinamento em esteira (com ou sem SPC) com outro treinamento orientado à tarefa da marcha $(15,17,19,21,24,25)$. O estudo de Visitin et al. (13) comparou o treinamento em esteira com SPC com treinamento sem SPC, e outros três estudos usaram intervenções não orientadas à tarefa como comparação $(16,20,23)$. Dois estudos descreveram a intervenção do grupo controle como sendo um treinamento simulado, ou placebo, sendo que Dean et al. (22) treinou o membro superior afetado, e Ada et al. (18) utilizou um treinamento de marcha domiciliar, orientado à tarefa em intensidade insuficiente para provocar efeito. Nenhum dos estudos encontrados comparou treinamento com SPC sem o uso da esteira com outra intervenção.

Em geral, os estudos encontrados mostraram que o treinamento de marcha em esteira com ou sem SPC tem efeito positivo sobre os parâmetros da marcha e a força muscular no membro inferior parético e no membro saudável. Estudos que utilizaram esteira rolante para reabilitar pacientes com sequela de AVE relataram melhora na velocidade de marcha (13-25), resistência à fadiga (distância e tempo de caminhada) $(13,16,18-22)$, cadência $(18,23,25)$, comprimento do passo e/ ou simetria $(18,23,25)$. Para Jaffe et al. (21), uma combinação e associação de treino de marcha, tanto na esteira como no solo, com trabalho de obstáculo, também melhorou os parâmetros de marcha, geralmente a velocidade. Os resultados dos estudos foram, em geral, baseados na velocidade de marcha e resistência, enquanto a dependência funcional da marcha ou outras medidas relevantes, como atividades de vida diária, qualidade de vida, ou ainda institucionalização, são relatadas em poucos estudos.

Uma hipótese clínica para explicar essa tendência ao favorecimento do treinamento em esteira e SPC como um tratamento é o fato de esta intervenção ser uma forma de aumentar a quantidade de prática. No estudo de Ada et al. (18), o aumento da prática é alcançado pela combinação de treinamento em esteira 
com outro exercício orientado à tarefa, como o treino de marcha em diferentes direções no solo e subir e descer escadas.

O treinamento em esteira com ou sem SPC parece ser tão efetivo quanto outras intervenções usadas como controle para pessoas com sequelas de AVE, sem evidências de ser prejudicial. Alguns aspectos específi$\cos$ da intervenção parecem ter impacto sobre a eficácia. Por exemplo, os efeitos do tratamento parecem estar relacionados com a dosagem do protocolo de treinamento utilizado. Nos estudos encontrados, o protocolo variou em intensidade, duração e frequência da caminhada na esteira, como pode ser visto na Tabela 1 . Os estudos relataram intervenções de duas semanas até seis meses, todas com resultados positivos. A frequência mínima foi de três vezes na semana, chegando a cinco em alguns estudos. Para Pohl et al. (23), um protocolo com uma progressão mais intensa da velocidade da esteira mostrou ser mais eficaz nos ganhos de velocidade da marcha, comparado com progressões de velocidade limitadas.

As características dos sujeitos também pareceram ser importantes na eficácia das intervenções. Dos estudos encontrados, dois realizaram treinamento em esteira em sujeitos agudos com no máximo 30 dias do início dos sintomas $(17,19)$, cinco incluíram pacientes transcorridos de 30 dias a três meses $(13,15,16,20$, 23) após o AVE, um estudo incluiu pacientes com mais de três meses de AVE (22), dois incluíram pacientes crônicos, ou seja, mais de seis meses após o evento $(18,21)$, e outro não especificou o tempo de lesão dos pacientes (25). Os estudos com pacientes muito agudos podem comprometer a análise do efeito específico do treinamento, uma vez que não é possível afirmar quanto da melhora pode ser atribuída a uma recuperação espontânea da marcha no período agudo após AVE.

Ainda, de acordo com a Moseley et al. (26), indivíduos independentes na marcha poderiam se beneficiar mais com programas de treinamento em esteira, em comparação com indivíduos dependentes. Além disso, resultados de um ensaio clínico (13) mostraram que indivíduos dependentes são mais favorecidos se for utilizado o SPC durante o treinamento em esteira. Dessa forma, diferenciar o nível de dependência para a marcha pode ser importante na definição do desenho de um ensaio clínico eficiente.

Uma meta-análise (26) sobre o uso da esteira e SPC na recuperação da marcha após um AVE concluiu que não houve diferenças entre o treinamento em esteira com ou sem SPC e outras intervenções na velocidade de marcha, embora uma análise secundária tenha mostrado que, entre as pessoas que deambulavam independentemente no início do tratamento, o uso da esteira foi eficaz para melhorar a velocidade de marcha. De acordo com a revisão, existe ainda uma necessidade urgente de estudos em larga escala, bem desenhados para avaliar os efeitos do treinamento em esteira com e sem SPC na marcha após um AVE, uma vez que os estudos ainda não são conclusivos quanto à superioridade em comparação com outros tratamentos convencionais, e existe hoje um grande marketing sobre esta prática.

Tabela 1 - Resumo dos testes de avaliação utilizados e dosagem das intervenções nos principais estudos de treinamento de marcha em esteira

(continua)

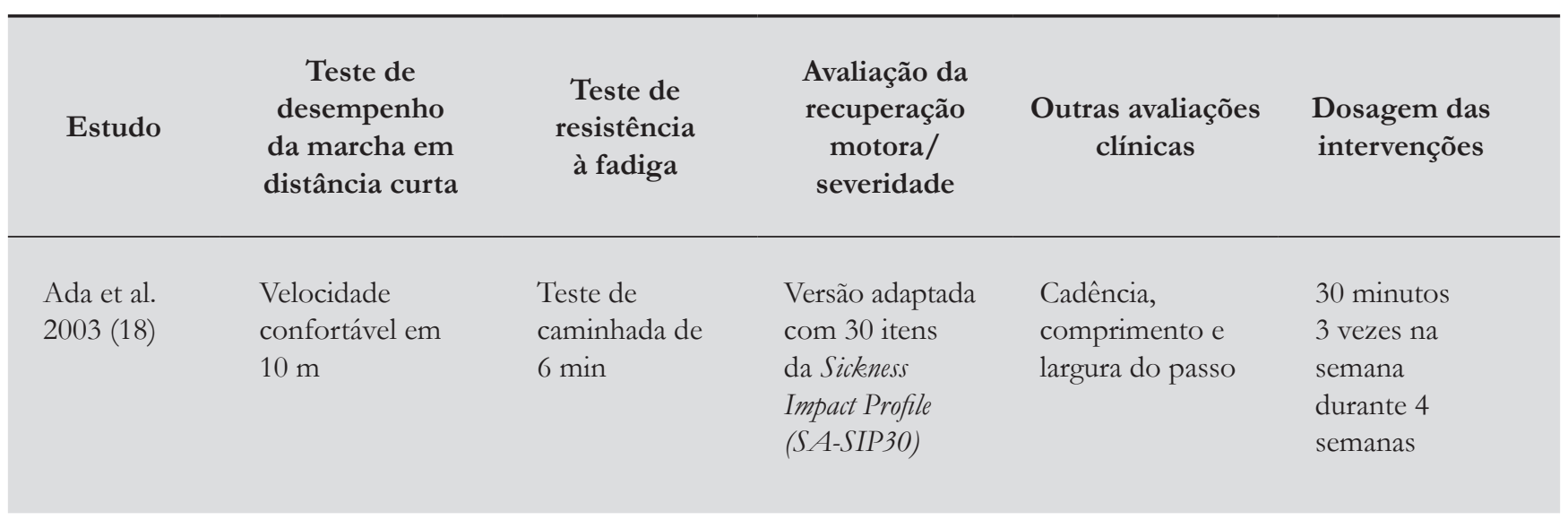


Tabela 1 - Resumo dos testes de avaliação utilizados e dosagem das intervenções nos principais estudos de treinamento de marcha em esteira

\begin{tabular}{|c|c|c|c|c|c|}
\hline Estudo & $\begin{array}{c}\text { Teste de } \\
\text { desempenho } \\
\text { da marcha em } \\
\text { distância curta }\end{array}$ & $\begin{array}{l}\text { Teste de } \\
\text { resistência } \\
\text { à fadiga }\end{array}$ & $\begin{array}{l}\text { Avaliação da } \\
\text { recuperação } \\
\text { motora/ } \\
\text { severidade }\end{array}$ & $\begin{array}{c}\text { Outras avaliações } \\
\text { clínicas }\end{array}$ & $\begin{array}{l}\text { Dosagem das } \\
\text { intervenções }\end{array}$ \\
\hline $\begin{array}{l}\text { da Cunha et al. } \\
2002(19)\end{array}$ & $\begin{array}{l}\text { Velocidade rápida } \\
\text { de marcha em } 5 \mathrm{~m}\end{array}$ & $\begin{array}{l}\text { Distância } \\
\text { máxima } \\
\text { percorrida em } \\
5 \text { min }\end{array}$ & $\begin{array}{l}\text { National Institutes } \\
\text { of Health (NIH) } \\
\text { Stroke Scale }\end{array}$ & $\begin{array}{l}\text { Functional } \\
\text { Ambulation } \\
\text { Category (FAC), } \\
\text { Medida de } \\
\text { Independência } \\
\text { funcional (MIF) }\end{array}$ & $\begin{array}{l}20 \text { minutos, } \\
5 \text { vezes na } \\
\text { semana, durante } \\
2 \text { a } 3 \text { semanas }\end{array}$ \\
\hline $\begin{array}{l}\text { Dean et al. } \\
2000(22)\end{array}$ & $\begin{array}{l}\text { Velocidade } \\
\text { confortável em } \\
10 \mathrm{~m}\end{array}$ & $\begin{array}{l}\text { Teste de } \\
\text { caminhada de } \\
6 \text { min }\end{array}$ & $\longrightarrow$ & $\begin{array}{l}\text { Tempo de } \\
\text { levantar e andar, } \\
\text { Step Test }\end{array}$ & $\begin{array}{l}60 \text { minutos de } \\
\text { intervenção, } \\
\text { sendo } 5 \text { minutos } \\
\text { na esteira, } \\
3 \text { vezes na } \\
\text { semana, durante } \\
4 \text { semanas }\end{array}$ \\
\hline $\begin{array}{l}\text { Eich et al. } \\
2004 \text { (20) }\end{array}$ & $\begin{array}{l}\text { Velocidade rápida } \\
\text { em } 10 \mathrm{~m}\end{array}$ & $\begin{array}{l}\text { Teste de } \\
\text { caminhada de } \\
6 \text { min }\end{array}$ & $\begin{array}{l}\text { Rivermead motor } \\
\text { asessement Scale }\end{array}$ & $\begin{array}{l}\text { Qualidade da } \\
\text { marcha com o } \\
\text { Rancho los Amigos } \\
\text { gait analysis }\end{array}$ & $\begin{array}{l}30 \text { minutos, } \\
5 \text { vezes na } \\
\text { semana, durante } \\
6 \text { semanas }\end{array}$ \\
\hline $\begin{array}{l}\text { Jaffe et al. } \\
2004 \text { (21) }\end{array}$ & $\begin{array}{l}\text { Velocidade } \\
\text { confortável e } \\
\text { rápida em } 6 \mathrm{~m}\end{array}$ & $\begin{array}{l}\text { Teste de } \\
\text { caminhada de } \\
6 \text { min }\end{array}$ & $\longrightarrow$ & $\begin{array}{l}\text { Habilidade ao } \\
\text { cruzar obstáculos }\end{array}$ & $\begin{array}{l}60 \text { minutos, } \\
3 \text { vezes na } \\
\text { semana, durante } \\
2 \text { semanas }\end{array}$ \\
\hline $\begin{array}{l}\text { Kosak et al. } \\
2000(16)\end{array}$ & $\begin{array}{l}\text { Velocidade } \\
\text { confortável em } \\
2 \text { min }\end{array}$ & $\begin{array}{l}\text { Distância } \\
\text { percorrida em } \\
\text { velocidade } \\
\text { confortável } \\
\text { até que o } \\
\text { participante não } \\
\text { mais tolerasse }\end{array}$ & & MIF & $\begin{array}{l}45 \text { minutos, } \\
5 \text { vezes na } \\
\text { semana, durante } \\
2 \text { a } 3 \text { semanas }\end{array}$ \\
\hline $\begin{array}{l}\text { Laufer et al. } \\
2001 \text { (15) }\end{array}$ & $\begin{array}{l}\text { Velocidade rápida } \\
\text { de marcha em } \\
10 \mathrm{~m}\end{array}$ & $\longrightarrow$ & $\longrightarrow$ & $\begin{array}{l}\text { FAC, Standing } \\
\text { Balance Test }\end{array}$ & $\begin{array}{l}8 \text { a } 20 \text { minutos, } \\
5 \text { vezes na } \\
\text { semana, durante } \\
3 \text { semanas }\end{array}$ \\
\hline
\end{tabular}


Tabela 1 - Resumo dos testes de avaliação utilizados e dosagem das intervenções nos principais estudos de treinamento de marcha em esteira

(conclusão)

\begin{tabular}{|c|c|c|c|c|c|}
\hline Estudo & $\begin{array}{c}\text { Teste de } \\
\text { desempenho } \\
\text { da marcha em } \\
\text { distância curta }\end{array}$ & $\begin{array}{l}\text { Teste de } \\
\text { resistência } \\
\text { à fadiga }\end{array}$ & $\begin{array}{c}\text { Avaliação da } \\
\text { recuperação } \\
\text { motora/ } \\
\text { severidade }\end{array}$ & $\begin{array}{l}\text { Outras avaliações } \\
\text { clínicas }\end{array}$ & $\begin{array}{l}\text { Dosagem das } \\
\text { intervenções }\end{array}$ \\
\hline $\begin{array}{l}\text { Liston et al, } \\
2000 \text { (25) }\end{array}$ & $\begin{array}{l}\text { Velocidade } \\
\text { confortável em } \\
10 \mathrm{~m}\end{array}$ & $\longrightarrow$ & $\longrightarrow$ & $\begin{array}{l}\text { Cadência, } \\
\text { Comprimento } \\
\text { do passo, teste de } \\
\text { sentar e levantar, } \\
\text { Nottingham } \\
\text { Extended } A D L \\
\text { Scale (avaliação } \\
\text { de atividades de } \\
\text { vida diária) }\end{array}$ & $\begin{array}{l}60 \text { minutos, } \\
3 \text { vezes na } \\
\text { semana, durante } \\
4 \text { semanas }\end{array}$ \\
\hline $\begin{array}{l}\text { Nilson el al. } \\
2001 \text { (17) }\end{array}$ & $\begin{array}{l}\text { Velocidade } \\
\text { confortável em } \\
10 \mathrm{~m}\end{array}$ & & $\begin{array}{l}\text { Escala de } \\
\text { Avaliação de } \\
\text { Fugl-Meyer }\end{array}$ & Berg, FAC, MIF & $\begin{array}{l}30 \text { minutos, } \\
5 \text { vezes na } \\
\text { semana, durante } \\
9 \text { a } 10 \text { semanas }\end{array}$ \\
\hline $\begin{array}{l}\text { Pohl et al. } \\
2002 \text { (23) }\end{array}$ & $\begin{array}{l}\text { Velocidade } \\
\text { confortável em } \\
10 \mathrm{~m}\end{array}$ & $\longrightarrow$ & $\longrightarrow$ & $\begin{array}{l}\text { Cadência, } \\
\text { Comprimento do } \\
\text { passo, FAC }\end{array}$ & $\begin{array}{l}30 \text { minutos, } \\
3 \text { vezes na } \\
\text { semana, durante } \\
4 \text { semanas }\end{array}$ \\
\hline $\begin{array}{l}\text { Visitin et al. } \\
1998 \text { (13) }\end{array}$ & $\begin{array}{l}\text { Distância } \\
\text { percorrida em } 3 \mathrm{~m}\end{array}$ & $\begin{array}{l}\text { Resistência } \\
\text { ercorrendo } \\
10 \mathrm{~m} \text { em } \\
\text { vai-e-vem até } \\
\text { exaustão }\end{array}$ & $\begin{array}{l}\text { The Stroke } \\
\text { Rehabilitation } \\
\text { Assessment } \\
\text { of Movement } \\
\text { (STREAM) }\end{array}$ & Berg & $\begin{array}{l}20 \text { minutos, } \\
4 \text { vezes na } \\
\text { semana, durante } \\
6 \text { semanas }\end{array}$ \\
\hline $\begin{array}{l}\text { Werner et al. } \\
2002(24)\end{array}$ & $\begin{array}{l}\text { Velocidade rápida } \\
\text { de marcha em } \\
10 \mathrm{~m}\end{array}$ & $\longrightarrow$ & $\begin{array}{l}\text { Rivermead motor } \\
\text { asessement Scale }\end{array}$ & $\begin{array}{l}\text { FAC, } \\
\text { espasticidade de } \\
\text { flexores plantares }\end{array}$ & $\begin{array}{l}15 \text { a } 20 \text { minutos, } \\
5 \text { vezes na } \\
\text { semana, durante } \\
2 \text { semanas }\end{array}$ \\
\hline
\end{tabular}

\section{Treinamento cardiorrespiratório}

A capacidade aeróbica de pacientes hemiparéticos é consideravelmente diminuída, com valores de $\mathrm{VO}_{2 \mathrm{MAX}}$ que variam de $50 \%$ a $75 \%$ do esperado, quando comparados com indivíduos saudáveis de mesma idade. Esse decréscimo da aptidão física está relacionado a diversos fatores, tais como comorbidades 
cardiovasculares associadas ao AVE, imobilidade, diminuição da capacidade de locomoção e déficits motores (8).

Estudos têm demonstrado que o treinamento com ênfase no condicionamento cardiopulmonar melhora o $\mathrm{VO}_{2 \mathrm{MAx}}$ ou $\mathrm{VO}_{2}$ de pico e diminui a frequência cardíaca (FC) de repouso em indivíduos hemiparéticos, além de reduzir o consumo de energia durante um teste de esforço (27). O principal objetivo dos programas de treinamento aeróbico é a melhora da aptidão cardiopulmonar, que está consideravelmente diminuída em indivíduos com hemiparesia. Os principais parâmetros determinantes são a melhora $\mathrm{VO}_{2 \mathrm{MAX}}$ ou do $\mathrm{VO}_{2}$ de pico e a potência máxima.

Foram encontrados poucos ensaios clínicos randomizados que investigaram o efeito do treinamento aeróbico especificamente sobre a aptidão cardiopulmonar, tendo com medida de desfecho principal o $\mathrm{VO}_{2 \mathrm{MAX}}$ ou $\mathrm{VO}_{2}$ de pico para indicar a capacidade aeróbica em indivíduos após AVE (27-31). Nesses estudos a avaliação inicial consistiu num teste de esforço no cicloergômetro $(27,30,31)$ ou em esteira rolante $(28,29)$, o que permitiu a prescrição individual de treinamento e determinou os parâmetros cardiorrespiratórios de base $\left(\mathrm{VO}_{2 \mathrm{MAX}}, \mathrm{VO}_{2}\right.$ de pico, frequência cardíaca máxima e de repouso, pressão arterial). Nos estudos encontrados, as características dos sujeitos e o tempo decorrido desde o evento cerebrovascular variaram, assim como a duração e intensidade dos programas. A Tabela 2 apresenta um resumo das intervenções e principais resultados dos estudos encontrados.

Dos estudos citados, o que teve menor duração foi o estudo de Chu et al. (31), num total de oito semanas de intervenção em 13 indivíduos crônicos (1 ano após o AVE no mínimo), com exercícios aeróbicos aquáticos com imersão na altura do peito. A intensidade utilizada foi $80 \%$ da FC de reserva. O grupo controle realizou um programa com exercícios para membros superiores na posição sentada. $\mathrm{O}$ programa mostrou ser benéfico na melhora da aptidão cardiorrespiratória, com melhoras no $\mathrm{VO}_{2}$ de pico de $22,5 \%$, além de melhorar a velocidade de marcha confortável $(9,2 \%)$.

O estudo de Potempa et al. (27), totalizou dez semanas de intervenção. Participaram 42 sujeitos crônicos (mais de seis meses), com hemiparesia leve a moderada, que realizaram exercícios aeróbicos em cicloergômetro, revelando melhoras do $\mathrm{VO}_{2 \mathrm{MAX}}$ de $0 \%$ a 35,7\%. O grupo controle recebeu exercícios com movimentos passivos. Os autores concluíram que, além de melhorar a capacidade aeróbica e a resposta da pressão arterial sistólica submáxima durante o treinamento, existe uma relação entre a melhora da capacidade aeróbica e da função sensório-motora, o que pode indicar que o treinamento aeróbico em bicicleta beneficia funcionalmente indivíduos capazes de treinar em intensidade que promova melhoramento da capacidade aeróbica.

O estudo de Macko et al. (29) em 61 pacientes após AVE crônico (acima de seis meses após o AVE), com treinamento aeróbico em esteira, mostrou uma melhora de $17 \%$ na aptidão cardiopulmonar, enquanto no grupo controle com um programa de reabilitação convencional, com caminhada de baixa intensidade e alongamentos, não houve melhoras na aptidão cardiorrespiratória. Os dois grupos receberam o mesmo tempo de intervenção a cada sessão. O grupo controle melhorou a velocidade de marcha em curta distância, porém não foi capaz de melhorar o desempenho em testes de resistência, como o teste de caminhada de seis minutos. De acordo com os autores, os resultados do estudo demonstram os benefícios fisiológicos e funcionais de um treinamento orientado à tarefa administrado em forma de exercício aeróbico progressivo. Os resultados sugerem que prescrições de treinamento com ênfase na progressão da velocidade, em vez da duração, poderão resultar em ganhos na aptidão física, além da função locomotora.

O estudo de Duncan et al. (30) avaliou os efeitos do treinamento aeróbico em uma população de cem indivíduos agudos, transcorridos de 30 a 150 dias após o AVE. A intervenção baseou-se em um programa de exercícios em casa supervisionados por fisioterapeutas, incluindo exercícios de alongamento, fortalecimento e de equilíbrio, exercícios em cicloergômetro de 25 a 30 minutos a 40 revoluções por minuto, e resistência tolerada pelo pacientes. A duração total de cada sessão foi de 90 minutos. O grupo controle recebeu cuidado usual, ou seja, fisioterapia convencional, conforme prescrita por seus fisioterapeutas, embora $46 \%$ dos participantes não tenham recebido tratamento algum. Os indivíduos apresentavam déficits de leve a moderado, como no estudo de Potempa et al. (27). Os indivíduos do grupo experimental tiveram uma melhora no $\mathrm{VO}_{2}$ de pico de $9 \%$, além de melhorar em $25,7 \%$ a velocidade de marcha em 10 metros e em $25,9 \%$ a resistência para caminhada no teste de caminhada de seis minutos, enquanto o grupo controle só foi capaz de melhorar a velocidade de marcha em 10 metros. 
O estudo de Katz-Leurer et al. (32), embora não tenha avaliado o $\mathrm{VO}_{2 \mathrm{MAx}}$ pré e pós intervenção, propôs um programa de treinamento aeróbico com cicloergômetro em um estágio muito agudo após o AVE, de 48 horas até 30 dias após o início dos sinais clínicos do primeiro AVE. A intensidade de treinamento variou dependendo da tolerância do sujeito e não ultrapassou $60 \%$ da frequência cardíaca de reserva. Embora não esteja relatado o tratamento recebido pelo grupo controle, os autores demonstraram que o exercício aeróbico iniciado em fase aguda promoveu melhoras na capacidade aeróbica e na habilidade funcional em pacientes moderadamente comprometidos, além disso, reduziu fatores relacionados a risco cardiovascular, tais como a pressão arterial e a frequência cardíaca de repouso.

Com base os estudos encontrados, é possível observar que o treinamento aeróbico pode melhorar a aptidão cardiorrespiratória em indivíduos após AVE, tanto em estágio muito agudo como crônico. Desta forma, seria possível assumir que programas que tenham duração de no mínimo oito semanas, com prática mínima de 30 minutos, três vezes na semana, em intensidades próximas ou acima de $60 \%$ da FC de reserva, sejam benéficos e possam ser introduzidos na prática clínica, sem evidência de ser prejudicial em uma população sem doenças cardiovasculares graves associadas.

Embora os protocolos de exercícios descritos nos estudos estejam de acordo com as recomendações da American College of Sports Medicine (ACSM) para a melhora da aptidão cardiorrespiratória, é importante ressaltar que tais diretrizes são estabelecidas para indivíduos saudáveis. Não se sabe se exercícios com intensidades mais baixas poderiam ser suficientes para melhorar a aptidão cardiorrespiratória em indivíduos acometidos por AVE, muitos dos quais apresentam descondicionamento severo (8). Exercícios aeróbicos em intensidades de treinamento baixas, a partir de 30\% do consumo de oxigênio, foram efetivos para melhorar a aptidão cardiovascular em indivíduos menos condicionados, embora saudáveis (33). Ainda foi demonstrado que pequenas sessões cumulativas de treinamento aeróbico produziram os mesmos efeitos de longas sessões de exercício (34). Não se sabe, no entanto, até que ponto tal princípio é verdadeiro em indivíduos com hemiparesia.

Um estudo (35) relatou um treinamento incluindo períodos de curtas sessões de exercício, com dois a três minutos de caminhada em esteira, intercaladas com períodos de repouso de mesma duração, totalizando 40 minutos, três vezes na semana, em pacientes com hemiparesia crônica com baixa aptidão física. Resultados positivos, com aumento do $\mathrm{VO}_{2}$ de pico, redução do custo energético durante a marcha, e aumento na capacidade de carga de trabalho foram relatados, e os autores ainda sugeriram que tais ganhos foram favoráveis para aumentar a mobilidade funcional de indivíduos crônicos. No entanto, o estudo não teve um grupo controle. A resistência aeróbica é um déficit importante em indivíduos após AVE, e se pequenas sessões de atividade são igualmente efetivas, poderia ser uma maneira excelente de treinar a resistência aeróbica nessa população.

Algumas limitações puderam ser identificadas nos estudos revisados. O estudo de Duncan et al. (30), por exemplo, cita como limitação o fato de não poder responder se uma menor intensidade de exercício teria sido tão efetiva quanto a usada no seu treinamento, além de não ser possível determinar qual componente do programa (estrutura, progressão ou intensidade) contribuiu para os resultados atingidos. Outra questão importante está no fato de que alguns estudos terem usado a mesma tarefa para avaliar e treinar a aptidão cardiorrespiratória $(27,29,30,32)$ e assim não se pode excluir a possibilidade de a melhora no desempenho no teste de exercício pós-treinamento ter sido em função do efeito da prática, mais do que uma verdadeira adaptação cardiorrespiratória. Outra limitação encontrada seria o relato do uso de certas medicações, como beta-bloqueadores, que podem alterar a resposta cardiovascular ao exercício; falhas ao relatar as informações sobre a medicação podem diminuir a qualidade de interpretação de alguns estudos. Apenas um estudo (27) relatou mudanças na medicação no decorrer da intervenção. Além disso, nos estudos revisados os indivíduos apresentaram um único AVE isquêmico ou hemorrágico, e comprometimento motor leve ou moderado, não apresentando patologias cardiovasculares severas ou instáveis associadas; desta forma, o efeito de exercícios aeróbicos em populações com comprometimentos severos e altos riscos cardíacos ainda necessita ser explorado.

Em virtude do número limitado de sujeitos na maioria dos estudos, com leve a moderado comprometimento motor, e ausência de patologias cardíacas severas, é preciso ter cautela ao generalizar esses resultados para a comunidade de indivíduos com hemiparesia. Igualmente, o efeito do treinamento em longo prazo ou um acompanhamento do efeito do condicionamento aeróbico mesmo após o término do programa também necessita ser mais explorado. 
Tabela 2 - Resumo dos estudos envolvendo condicionamento cardiorrespiratório

\begin{tabular}{|c|c|c|c|}
\hline Estudo & Tipo de treinamento & Dosagem & Resultados \\
\hline $\begin{array}{l}\text { Chu et al. } \\
2004 \text { (31) }\end{array}$ & $\begin{array}{l}\text { Experimental: exercícios } \\
\text { aquáticos de resistência } \\
\text { aeróbica. } \\
\text { Controle: tratamento não é } \\
\text { mencionado. }\end{array}$ & $\begin{array}{l}\text { Exercício em intensidade de } \\
\text { até } 80 \% \text { da FC máxima } \\
\text { Duração total da atividade } \\
\text { de } 1 \text { hora, sendo } 30 \text { min de } \\
\text { atividades aeróbicas } 3 \text { vezes } \\
\text { na semana durante } 8 \text { semanas. }\end{array}$ & $\begin{array}{l}\text { Melhora significativa em relação } \\
\text { ao grupo controle: } \\
\mathrm{VO}_{2} \text { de pico }(\uparrow 22,5 \%) \\
\text { Carga de trabalho de pico }(\uparrow 9,4 \%) \\
\text { Velocidade de marcha auto } \\
\text { selecionada }(\uparrow 9,2 \%) \text {. }\end{array}$ \\
\hline $\begin{array}{l}\text { Duncan et al. } \\
2003(30)\end{array}$ & $\begin{array}{l}\text { Experimental: programa } \\
\text { domiciliar supervisionado } \\
\text { incluindo atividade aeróbica } \\
\text { com cicloergômetro. } \\
\text { Grupo controle: } \\
\text { fisioterapia convencional. }\end{array}$ & $\begin{array}{l}\text { A duração total da atividade } \\
\text { de } 90 \text { minutos, destes } 25 \text { a } \\
30 \text { minutos de atividade em } \\
\text { cicloergômetro a } 40 \mathrm{rpm}, 3 \\
\text { vezes por semana durante } \\
36 \text { sessões. }\end{array}$ & $\begin{array}{l}\text { Melhoras significativas em relação } \\
\text { ao grupo controle: } \\
\mathrm{VO}_{2} \text { de pico }(\uparrow 9 \%) \\
\mathrm{TC} \text { de } 6 \text { min }(\uparrow 26 \%) \\
\text { Velocidade de marcha auto } \\
\text { selecionada }(\uparrow 26 \%) \text {. }\end{array}$ \\
\hline $\begin{array}{l}\text { Katz-Leurer et al. } \\
2003 \text { (32) }\end{array}$ & $\begin{array}{l}\text { Experimental: } \\
\text { exercício aeróbico em } \\
\text { cicloergômetro. } \\
\text { Controle: tratamento não } \\
\text { mencionado }\end{array}$ & $\begin{array}{l}\text { Exercício em intensidade de } \\
\text { até } 60 \% \text { da FC máxima } \\
\text { Duração de até } 20 \mathrm{~min} \\
\text { (semana1-2), até } 30 \mathrm{~min} \\
\text { (semana 3-8) } 3 \text { vezes na } \\
\text { semana durante } 8 \text { semanas. }\end{array}$ & $\begin{array}{l}\text { Melhoras os seguintes parâmetros: } \\
\text { Carga de trabalho de pico } \\
\text { ( } \uparrow 176,9 \%) \text {, número de degraus } \\
\text { percorridos até fadigar, velocidade } \\
\text { de marcha auto selecionada, MIF. }\end{array}$ \\
\hline $\begin{array}{l}\text { Macko et al. } \\
2005 \text { (29) }\end{array}$ & $\begin{array}{l}\text { Experimental: Treinamento } \\
\text { aeróbico na esteira. } \\
\text { Controle: programa de } \\
\text { reabilitação convencional } \\
\text { com caminhada de baixa } \\
\text { intensidade e alongamentos. }\end{array}$ & $\begin{array}{l}\text { Intensidade de } 60 \text { a } 70 \% \text { da } \\
\text { FC de reserva, } 3 \text { vezes na } \\
\text { semana ( } 40 \text { min de duração) } \\
\text { durante } 6 \text { meses. }\end{array}$ & $\begin{array}{l}\text { Melhoras significativas no } \mathrm{VO}_{2} \\
\text { de pico }(\uparrow 17 \%) \text {, no TC de } 6 \mathrm{~min} \\
(30 \%) \text { e mobilidade funcional }(56 \%) \text {. }\end{array}$ \\
\hline $\begin{array}{l}\text { Potempa et al. } \\
1995 \text { (27) }\end{array}$ & $\begin{array}{l}\text { Experimental: } \\
\text { exercício aeróbico em } \\
\text { cicloergômetro. } \\
\text { Controle: exercícios } \\
\text { passivos de movimentação } \\
\text { articular. }\end{array}$ & $\begin{array}{l}\text { Da semana } 1-4: 30-50 \% \text { da } \\
\text { carga de trabalho máxima, } \\
\uparrow \text { de acordo com a tolerância; } \\
\text { manutenção da carga máxima } \\
\text { alcançada nas semanas 5-10, } \\
\text { realizado } 3 \text { vezes na semana } \\
\text { (duração de } 30 \text { min), durante } \\
10 \text { semanas. }\end{array}$ & $\begin{array}{l}\text { Melhora significativa em relação ao } \\
\text { grupo controle: } \\
\mathrm{VO}_{2} \text { de pico }(\uparrow 13,3 \%) \\
\text { Carga de trabalho de pico }(\uparrow 44,3 \%) \\
\text { Ventilação minuto de pico. }\end{array}$ \\
\hline
\end{tabular}

\section{Treinamento de fortalecimento muscular para membros inferiores}

A fraqueza muscular é um comprometimento motor comum em pacientes após um AVE (9). As características da força muscular que seguem o AVE incluem redução na geração de torque isométrico e isocinético, além de lentidão para gerar o torque $(9,36)$. Tal inabilidade para gerar torque demonstrou estar 
relacionada com o desempenho em diversas tarefas funcionais, tais como transferências, levantar a partir de sentado, velocidade de marcha e desempenho ao subir escadas, sugerindo que o treinamento de força poderia levar a uma melhora no desempenho funcional (36).

Embora o uso de exercícios com resistência seja comumente aceito como um excelente método de treinamento de força em músculos sadios, até bem pouco tempo ainda existia polêmica a respeito dos benefícios do exercício resistido em pacientes hipertônicos espásticos (36). Apesar da restrição de muitos fisioterapeutas quanto ao uso de técnicas de fortalecimento muscular, existem hoje estudos mostrando que o fortalecimento muscular apresenta benefícios na reabilitação de indivíduos após AVE (36-41).

Foram encontrados seis estudos (9,37-41) que detalham protocolos de fortalecimento muscular para os membros inferiores com exercícios isocinéticos, isométricos e isotônicos. Os protocolos frequentemente usados são comparados com programas de treinamento propostos para idosos, isto é, exercícios com carga de no mínimo 50\% da carga máxima, com 8-12 repetições. A Tabela 3 apresenta um resumo das intervenções e principais resultados dos estudos encontrados.

No estudo de Oullette et al. (38), o grupo experimental recebeu um programa de treinamento resistido com carga de intensidade de 70\% de 1 RM. Os sujeitos realizaram leg press bilateral, extensão de joelho unilateral nos dois membros, dorsiflexão e plantiflexão unilateral, três vezes na semana, durante 12 semanas. O estudo de Kim et al. (39) teve uma duração menor: foram 6 semanas com 3 sessões de 45 minutos. A intervenção consistiu de 5 minutos de aquecimento, com movimentos ativos de flexão de extensão de quadril, joelho e tornozelo, 5 minutos de alongamentos do membro parético, e aproximadamente 30 minutos de exercícios de fortalecimento com máximo esforço concêntrico no dinamômetro isocinético Kin-Con, com 3 séries de 10 repetições de flexão e extensão de quadril, joelho e tornozelo. Sharp e Brouwer (8) realizaram um estudo de pré e pós-teste com 15 indivíduos, que realizaram treinamento de força isocinético por 6 semanas, 3 vezes na semana, durante 40 minutos. Foram encontradas diferenças significativas no pico de torque de extensores e flexores de joelho, velocidade de marcha e nível de atividade física no pós-teste. Este estudo foi importante porque mostrou que a espasticidade não aumenta com exercícios de fortalecimento. No entanto, a falta de um grupo controle limita a interpretação dos resultados, uma vez que pacientes após AVE, mesmo crônicos, podem melhorar sua condição com a prática de tarefas específicas (42).

Em outro estudo com pacientes crônicos sem grupo controle (41), foram avaliados os efeitos do treinamento de força progressivo, tanto no lado afetado como no não afetado. O programa consistiu de 12 semanas com duas sessões semanais, e os exercícios eram realizados com $70 \%$ de $1 \mathrm{RM}$. A força melhorou $68 \%$ no lado afetado e $48 \%$ no lado não afetado. O desempenho funcional mostrado por meio de testes como levantar de uma cadeira cinco vezes em sequência, Motor Assessment Scale, e escala de equilíbrio de Berg também melhorou, no entanto, não se pode descartar a possibilidade desta melhora ser resultado do fortalecimento do lado menos afetado. Mais uma vez, a falta de um grupo controle limita a interpretação do estudo.

O estudo de Teixeira Salmela et al. (37) combinou treinamento de força com exercício aeróbico durante 10 semanas, cada sessão com duração de 60 a 90 minutos, 3 vezes na semana em pacientes com hemiparesia crônica. Foram encontrados resultados positivos em termos de aumento de força muscular, qualidade de vida, nível geral de atividade física, velocidade de marcha e desempenho na subida de escadas. Embora o efeito isolado do treinamento de força não tenha sido determinado, o estudo foi importante para demonstrar o benefício de uma combinação de intervenções com ênfase no condicionamento cardiopulmonar e fortalecimento muscular nessa população.

Outro estudo de Yang e colaboradores (40) propôs, em um ensaio clínico randomizado, um treinamento de força progressivo com exercícios resistidos orientados à tarefa em sujeitos com sequelas crônicas de AVE. Tais atividades incluíram levantar e alcançar objetos em diferentes direções, levantar a partir de sentado de diferentes alturas de assentos, dar passos para frente, para trás e para os lados sobre blocos de várias alturas, ficar na ponta dos pés e descer (para fortalecimento de flexores plantares), tendo cada estação de exercícios durado cinco minutos. A progressão ocorreu pelo aumento do número de repetições e aumento de complexidade do exercício, tal como altura do assento ou dos blocos. Após quatro semanas, foram observadas melhoras significativas na força muscular de flexores e extensores de quadril, joelho e tornozelo, além de melhoras em habilidades funcionais, tais como velocidade de marcha, tempo de levantar e andar, teste de 
caminhada de seis minutos e tempo de subida de degraus. O grupo controle não realizou intervenção alguma, o que torna difícil determinar se um treinamento de força progressivo orientado à tarefa poderia ser tão eficaz ou mesmo superior a um treinamento de força convencional.

Todos os estudos aqui citados foram realizados com pacientes crônicos e relatam adaptações positivas com o treinamento de força. A duração dos programas envolvendo treinamento de força específico, com exceção do estudo orientado à tarefa, foi de seis a 12 semanas, em concordância com estudos de treinamento de força conduzidos em indivíduos saudáveis (43). Todos os estudos relatam efeitos positivos do treinamento de força em vários aspectos funcionais, tais como velocidade de marcha, habilidade ao subir escadas, levantar de uma cadeira e atividades de vida diária. Somente um estudo (9) avaliou o efeito do destreinamento, mostrando que as melhoras ainda eram evidentes após quatro semanas do término do programa, embora não significativas, comparadas com os resultados pré-treinamento, com exceção do músculo quadríceps a $30^{\circ} / \mathrm{s}$. Apenas dois estudos avaliaram o tônus muscular $(9,37)$ e nenhum deles relatou efeitos negativos do treinamento de força sobre a espasticidade.

Embora os estudos tenham demonstrado melhoras na força muscular (37-41), velocidade de marcha (37-41), qualidade de vida (37) e desempenho funcional (37-41), a diversidade de protocolos utilizados em um número restrito de estudos ainda torna difícil estabelecer recomendações gerais sobre a forma de realização e progressão desse tipo de exercício para pacientes hemiparéticos na prática clínica diária.

Finalmente, o efeito de treinamentos de longa duração em hemiplégicos após AVE (maior do que 12 semanas) ainda necessita ser determinado, uma vez que os estudos não relatam os efeitos de um treinamento por período superior a 12 semanas. Embora no hemiplégico não se conheça o efeito de um treinamento de força prolongado, em adultos idosos saudáveis, o treinamento de força por um período superior a 24 meses não levou a um platô (43) no ganho de força. A progressão e a continuidade de um treinamento de resistência poderiam, assim, ser um caminho proveitoso para promover a melhora na força e função em indivíduos com hemiparesia.

Considerando os achados da literatura, é possível afirmar que o treinamento de força certamente apresenta um papel importante para reverter a fraqueza após um AVE, muito embora não deva ser visto como uma substituição para o treinamento funcional. $O$ treinamento resistido pode ser um complemento importante ou auxiliar na reabilitação tradicional. No entanto, mais estudos são necessários para uma plena compreensão dos parâmetros específicos que possam promover resultados mais eficazes, com eficientes resultados funcionais. Além disso, o efeito da retenção dos ganhos de força, entre outras habilidades funcionais, após o término do programa, também necessita ser mais explorado.

Tabela 3 - Resumo dos estudos envolvendo fortalecimento muscular

(continua)

\begin{tabular}{|c|c|c|c|}
\hline Estudo & Tipo de treinamento & Dosagem & Resultados \\
\hline $\begin{array}{l}\text { Kim et al. } \\
2001 \text { (39) }\end{array}$ & $\begin{array}{l}\text { Experimental: Treinamento } \\
\text { de força isocinético } \\
\text { concêntrico usando o } \\
\text { aparelho Isocinético } \\
\text { Kin-Com. } \\
\text { Controle: movimentação } \\
\text { passiva no Kin-Com. }\end{array}$ & $\begin{array}{l}3 \text { séries de } 10 \text { repetições de } \\
\text { máximo esforço concêntrico } \\
\text { de flexão e extensão de } \\
\text { quadril, joelho e tornozelo, } \\
3 \text { vezes na semana durante } \\
6 \text { semanas }\end{array}$ & $\begin{array}{l}\text { Tendência a aumento de força no } \\
\text { grupo experimental em comparação } \\
\text { ao grupo controle }(p=0.06 \text { ) } \\
\text { Escores compostos de força (soma } \\
\text { do percentual de mudança dos } \\
\text { valores pré e pós treinamento) } \\
\text { aumentaram } 507 \% \pm 559 \text { no grupo } \\
\text { experimental e } 142 \% \pm 193 \text { no } \\
\text { grupo controle no membro parético. } \\
\text { Habilidade para subir escadas e } \\
\text { velocidade de marcha aumentou } \\
\text { em ambos os grupos, sem diferença } \\
\text { estatística entre eles. }\end{array}$ \\
\hline
\end{tabular}


Tabela 3 - Resumo dos estudos envolvendo fortalecimento muscular

\begin{tabular}{|c|c|c|c|}
\hline Estudo & Tipo de treinamento & Dosagem & Resultados \\
\hline $\begin{array}{l}\text { Oulette et al. } \\
2004 \text { (38) }\end{array}$ & $\begin{array}{l}\text { Programa de treinamento } \\
\text { de alta intensidade com } \\
\text { leg press bilateral, extensão } \\
\text { de joelho unilateral com os } \\
\text { dois membros inferiores, } \\
\text { exercícios de plantiflexão e } \\
\text { dorsiflexão de tornozelo. } \\
\text { Grupo Controle: exercícios } \\
\text { bilaterais de amplitude de } \\
\text { movimento e flexibilidade } \\
\text { de membros superiores. }\end{array}$ & $\begin{array}{l}3 \text { séries de } 8-10 \text { repetições } \\
\text { a } 70 \% \text { de } 1 \mathrm{RM} \\
3 \mathrm{x} \text { na semana, durante } \\
12 \text { semanas }\end{array}$ & $\begin{array}{l}\text { Melhora significativa de uma máxima } \\
\text { repetição no leg press, extensão de } \\
\text { joelho bilateral, sem mudanças } \\
\text { no grupo controle. Dorsiflexão e } \\
\text { plantiflexão de tornozelo parético } \\
\text { melhorou significativamente } \\
\text { comparado ao grupo controle. } \\
\text { Melhora no auto relato de função } \\
\text { e incapacidade sem mudanças no } \\
\text { grupo controle. }\end{array}$ \\
\hline $\begin{array}{l}\text { Sharp e Brouwer } \\
1997 \text { (9) }\end{array}$ & $\begin{array}{l}5 \text { min de aquecimento } \\
\text { em cicloergômetro, } \\
\text { alongamentos de quadríceps } \\
\text { e ísquios-tibiais. } \\
\text { Treinamento isocinético } \\
\text { concêntrico de quadríceps } \\
\text { e ísquios-tibiais. }\end{array}$ & $\begin{array}{l}\text { 6-8 repetições ao máximo } \\
\text { de esforço a } 30^{\circ}, 60^{\circ}, 20^{\circ} / \mathrm{s} \text {, } \\
3 \text { vezes na semana durante } \\
6 \text { semanas }\end{array}$ & $\begin{array}{l}\text { Ganhos significativos na força de } \\
\text { quadríceps e ísquios-tibiais nas } 3 \\
\text { velocidades, melhora na velocidade } \\
\text { de marcha, sem mudanças } \\
\text { significativas no TLA e habilidade } \\
\text { para subir degraus. }\end{array}$ \\
\hline $\begin{array}{l}\text { Teixeira-Salmela } \\
\text { et al. } 1999 \text { (37) }\end{array}$ & $\begin{array}{l}\text { Experimental: exercícios } \\
\text { isométricos, concêntricos } \\
\text { e excêntricos utilizando } \\
\text { caneleiras e theraband. } \\
\text { Controle: } 7 \text { dos } 13 \text { sujeitos } \\
\text { do estudo serviram de } \\
\text { controle, sendo avaliados, } \\
\text { e reavaliados após } 10 \\
\text { semanas, sem ter recebido } \\
\text { intervenção. }\end{array}$ & $\begin{array}{l}30 \text { minutos } 3 \text { vezes na } \\
\text { semana, } 3 \text { séries de } \\
10 \text { repetições para quadril, } \\
\text { joelho e tornozelo a } 50 \% \\
1 \text {-RM (aumentados para } \\
80 \% \text { após } 2 \text { semanas), com } \\
\text { reavaliação a cada } 2 \text { semanas. } \\
\text { Além do treinamento } \\
\text { de força, 5-10 min de } \\
\text { aquecimento, } 10-20 \text { min } \\
\text { de exercício aeróbico com } \\
\text { caminhada em esteira mais } \\
\text { stepping ou pedaladas a } 70 \% \\
\text { da FC max, resfriamento de } \\
\text { 5-10 min }\end{array}$ & $\begin{array}{l}42,3 \% \text { de aumento na força avaliada } \\
\text { através do torque concêntrico } \\
\text { isocinético a } 30^{\circ} \text { e } 60^{\circ} / \mathrm{s} 28 \% \text { de } \\
\text { aumento da velocidade de marcha } \\
37,4 \% \text { de aumento na habilidade } \\
\text { para subir escadas. Melhora de } \\
39,2 \% \text { nas habilidades para realizar } \\
\text { atividades diárias de acordo com o } \\
\text { escore ajustado de atividade (EAA) } \\
\text { do Perfil de atividade Humana } \\
\text { (PAH). Melhora nos escores da } \\
\text { qualidade de vida ( } 77,8 \% \text { ) } \\
\text { Através do Perfil de Saúde de } \\
\text { Nottingham. }\end{array}$ \\
\hline $\begin{array}{l}\text { Weiss et al. } \\
2000 \text { (41) }\end{array}$ & $\begin{array}{l}\text { Treinamento de força } \\
\text { concêntrico e excêntrico } \\
\text { usando aparelhos de } \\
\text { musculação. }\end{array}$ & $\begin{array}{l}3 \text { séries de } 8-10 \text { repetições } \\
\text { a } 70 \% \text { de } 1 \text { RM, } 2 \text { vezes na } \\
\text { semana, durante } 12 \text { semanas }\end{array}$ & $\begin{array}{l}\text { Ganhos de força de } 68 \% \text { no MI } \\
\text { parético e } 48 \% \text { no não afetado, } \\
\text { melhoras na flexão, extensão, } \\
\text { abdução de quadril, flexão / extensão } \\
\text { de joelho, melhora na habilidade } \\
\text { em subir escadas, velocidade de } \\
\text { marcha, recuperação motora } \\
\text { (Motor Assessmet Scale) e equilíbrio } \\
\text { (BERG). }\end{array}$ \\
\hline
\end{tabular}


Tabela 3 - Resumo dos estudos envolvendo fortalecimento muscular

(conclusão)

\begin{tabular}{|c|c|c|c|}
\hline Estudo & Tipo de treinamento & Dosagem & Resultados \\
\hline $\begin{array}{l}\text { Yang et al. } \\
2006 \text { (40) }\end{array}$ & $\begin{array}{l}\text { Experimental: Exercícios } \\
\text { resistidos orientados a } \\
\text { tarefa: levantar e alcançar } \\
\text { objetos em diferentes } \\
\text { direções, levantar a partir } \\
\text { de sentado de diferentes } \\
\text { alturas de assentos, dar } \\
\text { passos a frente, para trás e } \\
\text { para os lados sobre blocos } \\
\text { de várias alturas, ficar na } \\
\text { ponta dos pés e descer para } \\
\text { fortalecimento de flexores } \\
\text { plantares, sendo que cada } \\
\text { estação de exercícios teve } \\
\text { duração de } 5 \text { minutos. } \\
\text { Controles: sem intervenção }\end{array}$ & $\begin{array}{l}30 \text { min de exercícios } \\
3 \text { vezes na semana durante } \\
4 \text { semanas }\end{array}$ & $\begin{array}{l}\text { Força muscular melhorou no grupo } \\
\text { exp. No lado não afetado (de } 23,9 \% \\
\text { a } 36,5 \% \text { ) e parético (de } 10,1 \% \text { a } \\
77,9 \% \text { ). Melhoras em habilidades } \\
\text { funcionais, tais como velocidade } \\
\text { de marcha, tempo de levantar e } \\
\text { andar, teste de caminhada de } 6 \\
\text { minutos e tempo de subida de } \\
\text { degraus. Os ganho de força esteve } \\
\text { significativamente associado com } \\
\text { melhoras nos testes funcionais. }\end{array}$ \\
\hline
\end{tabular}

\section{Considerações finais}

Por meio desta análise de estudos que investigaram os efeitos de diversos programas de treinamento sobre pacientes com hemiparesia, foi possível detectar que os exercícios de treinamento de marcha, cardiorrespiratório e fortalecimento muscular melhoraram o desempenho funcional desses pacientes quanto ao desempenho na marcha (velocidade, resistência). No entanto, diferenças metodológicas entre os estudos, tais como o tamanho da amostra, grau de comprometimento motor, presença de comorbidades cardiovasculares, tempo decorrido após o AVE e técnica especifica de treinamento empregada, dificultam a determinação da melhor população alvo e o melhor protocolo de treinamento. Torna-se complexo averiguar e comparar a efetividade de programas específicos por causa da variabilidade nos critérios de avaliação e técnicas de mensuração. Apesar disso, a maioria dos estudos com os mais diversos programas de treinamento mostrou resultados positivos, o que demonstra um ponto favorável para as terapias atualmente empregadas. Os programas de treinamento de marcha em esteira com ou sem suspensão de peso corporal estão atualmente bastante favorecidos, pois muitos estudos já demonstraram seus benefícios.

Muitas questões permanecem sem resposta, especialmente sobre o efeito de técnicas específicas nos sistemas musculoesquelético, cardiovascular e neurológico. Observa-se a necessidade de estudos que comparem diferentes técnicas, pois em grande parte deles os grupos controles realizaram pouca atividade ou atividade alguma, o que torna difícil perceber o efeito real de tal técnica de exercício. Além disso, são necessárias mais pesquisas para determinar protocolos eficazes para treinar indivíduos com diferentes níveis de comprometimento motor, tempo de lesão e risco cardíaco, além de estabelecer os efeitos em longo prazo de tais intervenções, assim como as relações entre a melhora da força muscular, marcha e capacidade cardiorrespiratória com capacidade funcional, realização de atividades diárias, participação, qualidade de vida e dependência. 


\section{Referências}

1. Bonita R. Epidemiology of stroke. Lancet. 1992;339(8789):342-4.

2. Massaro A. Dia mundial do AVC: prevenção e tratamento adequado diminuem índice de seqüelas e melhoram qualidade de vida do paciente. Revista Nutrição em Pauta. 2005. [acesso 24 out. 2009]. Disponível em: http:// www.nutricaoempauta.com.br/lista_artigo.php?cod=235

3. Mayo NE, Wood-Dauphinee S, Ahmed S, Gordon C, Higgins J, McEwen S, et al. Disablement following stroke. Disabil Rehabil. 1999;21(5-6):258-68.

4. Dobkin BH. Clinical practice: rehabilitation after stroke. N Engl J Med. 2005;352(16):1677-84.

5. Carr, JH, Shepherd RB. A motor learning model for stroke rehabilitation. Physiotherapy. 1989;89:372-80.

6. Wade DT, Wood VA, Heller A. Walking after stroke: measurement and recovery over the first three months. Scand J Rehabil Med. 1987;19(1):25-30.

7. Goldie PA, Matyas TA, Evans OM. Deficit and change in gait velocity during rehabilitation after stroke. Arch Phys Med Rehabil. 1996;77(10):1074-82.

8. MacKay-Lyons MJ, Makrides L. Longitudinal changes in exercise capacity after stroke. Arch Phys Med Rehabil. 2004;85(10):1608-12.

9. Sharp SA, Brouwer BJ. Isokinetic strength training of the hemiparetic knee: effects on function and spasticity. Arch Phys Med Rehabil. 1997;78(11):1231-6.

10. Macko RF, Ivey FM, Forrester LW. Task oriented aerobic exercise in chronic hemiparetic stroke: training protocols and treatment effects. Top Stroke Rehabil. 2005;12(1):45-57.

11. Hesse S, Werner C. Poststroke motor dysfunction and spasticity: novel pharmacological and physical treatment strategies. CNS Drugs. 2003;17(15):1093-107.

12. Hesse S, Jahnke MT, Schaffrin A, Lucke D, Reiter F, Konrad M. Immediate effects of therapeutic facilitation on the gait of hemiparetic patients as compared with walking with and without a cane. Electroencephalogr Clin Neurophysiol. 1998;109(6):515-22.

13. Visintin M, Barbeau H, Korner-Bitensky N, Mayo NE. A new approach to retrain gait in stroke patients through body weight support and treadmill stimulation. Stroke. 1998;29(6):1122-8.

14. Teixeira da Cunha Filho I, Lim PAC, Qureshy H, Henson H, Monga T, Protas EJ. A comparison of regular rehabilitation and regular rehabilitation with supported treadmill ambulation training for acute stroke patients. J Rehab Res Dev. 2001;38:245-55.

15. Laufer Y, Dickstein R, Chefez Y, Marcovitz E. The effect of treadmill training on the ambulation of stroke survivors in the early stages of rehabilitation: a randomised study. J Rehabil Res Dev. 2001;38(1):69-78.

16. Kosak MC, Reding MJ. Comparison of partial body weight-supported treadmill gait training versus aggressive bracing walking post stroke. Neurorehabil Neural Repair. 2000;14(1):13-9.

17. Nilsson L, Carlsson J, Danielsson A, Fugl-Meyer A, Hellström K, Kristensen L. Walking training of patients with hemiparesis at an early stage after stroke: a comparison of walking training on a treadmill with body weight support and walking training on the ground. Clin Rehabil 2001; 15:515-27. Clin Rehabil. 2001;15(5):515-27.

18. Ada L, Dean C, Hall J, Bampton J, Crompton S. A treadmill and overgrown walking program improves walking in persons residing in the community after stroke: a placebo-controlled, randomized trial. Arch Phys Med Rehabil. 2003;84:1486-9. 
19. Da Cunha IT, Lim PA, Qureshy H, Henson H, Monga T, Protas EJ. Gait outcomes after acute stroke rehabilitation with supported treadmill ambulation training: a randomized controlled pilot study. Arch Phys Med Rehabil. 2002;83(9):1258-65.

20. Eich HJ, Mach H, Werner C, Hesse S. Aerobic treadmill plus Bobath walking training improves walking in subacute stroke: a randomized controlled trial. Clin Rehabil. 2004;18(6):640-51.

21. Jaffe DL, Brown DA, Pierson-Carey CD, Buckley EL, Lew HL. Stepping over obstacles to improve walking in individuals with poststroke hemiplegia. J Rehabil Res Dev. 2004;41(3A):283-92.

22. Dean CM, Richards CL, Malouin F. Task-related circuit training improves performance of locomotor tasks in chronic stroke: a randomized, controlled pilot trial. Arch Phys Med Rehabil. 2000;81(4):409-17.

23. Pohl M, Mehrlholz J, Ritschel C, Rückriem S. Speed-dependent treadmill training in ambulatory hemiparetic stroke patients, a randomized controlled trial. Stroke. 2002;33(2):553-8.

24. Werner C, Frankenberg S, Treig T, Konrad M, Hesse S. Treadmill training with partial body weight support and an electromechanical gait trainer for restoration of gait in subacute stroke patients. Stroke. 2002;33(12):2895-901.

25. Liston R, Mickelborough J, Harris B, Hann AW, Tallis RC. Conventional physiotherapy and treadmill re-training for higher-level gait disorders in cerebrovascular disease. Age Ageing. 2000;29(4):311-8.

26. Moseley AM, Stark A, Cameron ID, Pollock A. Treadmill training and body weight support for walking after stroke. Cochrane Database Syst Rev. 2005;(4):CD002840.

27. Potempa K, Lopez M, Braun LT, Szidon P, Fogg L, Tincknell T. Physiological outcomes of aerobic exercise training in hemiparetic stroke patients. Stroke. 1995;26:101-5.

28. Macko RF, DeSouza CA, Tretter LD, Silver KH, Smith GV, Anderson PA, et al. Treadmill aerobic exercise training reduces the energy expenditure and cardiovascular demands of hemiparetic gait in chronic stroke patients. Stroke. 1997;28(2):326-30.

29. Macko RF, Ivey FM, Forrester LW, Hanley D, Sorkin JD, Katzel LI, et al. Treadmill exercise rehabilitation improves ambulatory function and cardiovascular fitness in patients with chronic stroke: A randomized, controlled trial. Stroke. 2005;36(10):2206-11.

30. Duncan P, Studenski S, Richards L, Gollub S, Lai SM, Reker D, et al. Randomized clinical trial of therapeutic exercise in subacute stroke. Stroke. 2003;34(9):2173-80.

31. Chu KS, Eng JJ, Dawson AS, Harris JE, Ozkaplan A, Gylfadottir S. Water-based exercise for cardiovascular fitness in people with chronic stroke: a randomized controlled trial. Arch Phys Med Rehabil. 2004;85(6):870-4.

32. Katz-Leurer M, Shochina M, Carmeli E, Friedlander Y. The influence of early aerobic training on the functional capacity in patients with cerebrovascular accident at the subacute stage. Arch Phys Med Rehabil. 2003;84(11):1609-14.

33. Swain DP, Franklin BA. $\mathrm{VO}_{2}$ reserve and the minimal intensity for improving cardiorespiratory fitness. Med Sci Sports Exerc. 2002;34:152-57.

34. Debusk RF, Stenestrand U, Sheehan M, Haskell WL. Training effects of long versus short bouts of exercise in healthy subjects. Am J Cardiol. 1990;65(15):1010-3.

35. Macko RF, Smith GV, Dobrovolny L, Sorkin JD, Goldberg AP, Silver KH. Treadmill training improves fitness reserve in chronic stroke patients. Arch Phys Med Rehabil. 2001;82(7):879-84.

36. Morris SL, Dodd KJ, Morris ME. Outcomes of progressive resistance strength training following stroke: a systematic review. Clin Rehabil. 2004;18(1):27-39.

37. Teixeira-Salmela LF, Olney SJ, Nadeau S, Brouwer B. Muscle strengthening and physical conditioning to reduce impairment and disability in chronic stroke survivors. Arch Phys Med Rehabil. 1999;80(10):1211-8. 
38. Ouellette MM, Le Brasseur NK, Bean JF, Phillips E, Stein J, Frontera WR, et al. High-intensity resistance training improves muscle strength, self-reported function, and disability in long-term stroke survivors. Stroke. 2004;35(6):1404-9.

39. Kim CM, Eng JJ, Macintyre DL, Dawson AS. Effects of isokinetics strength training on walking in persons with stroke: a double-blind controlled pilot study. J Stroke Cerebrovasc Dis. 2001;10(6):265-73.

40. Yang YR, Wang RY, Lin KH, Chu MY, Chan RC. Task-oriented progressive resistance strength training improves muscle strength and functional performance in individuals with stroke. Clin Rehabil. 2006;20(10):860-70.

41. Weiss A, Suzuki T, Bean J et al. High intensity strength training improves strength and functional performance after stroke. Am J Phys Med Rehabil. 2000;79(4):369-76.

42. Schaechter JD: Motor rehabilitation and brain plasticity after hemiparetic stroke. Prog Neurobiol. 2004;73(1):61-72.

43. Porter MM. Resistance training recommendations for older adults. Top Geriatr Rehabil. 2000;15(3):60-9.

Recebido: 07/11/2009

Received: 11/07/2009

Aprovado: $18 / 03 / 2010$

Approved: 03/18/2010

Revisado: 12/05/2010

Reviewed: 05/12/2010 